\title{
Tratamiento con insulina aspártica bifásica en pacientes con diabetes en mal control metabólico. Experiencia clínica programada
}

\author{
Manuel G arcía de los Ríos ${ }^{1}$, Carlos Wolff ${ }^{1 a}$, Erika D uarte ${ }^{2 b}$. \\ Biphasic insulin aspart for the \\ management of diabetic patients \\ with unsatisfactory metabolic control
}

Background: Biophasic insulin aspart (InAsBi) is a mixture of $30 \%$ of rapid acting soluble aspart insulin and $70 \%$ aspart insulin retarded with protamine. The soluble portion reduces postprandial serum glucose rises and the retarded portion reduces basal glucose levels. Aim: To assess the efficacy of biphasic insulin aspart in diabetics with a bad metabolic control. Material and methods: Multicentric study that included diabetic patients with a glycosilated hemoglobin over $7 \%$ that were transferred to treatment with InAsBi, given in one to three daily doses, according to glycemic control and followed for 12 weeks. At the end of follow up, glycosilated hemoglobin levels (HbA1c) were measured again. Results: One hundred ninety six patients were enrolled and 154, age 59 \pm 12 (84 females), completed the follow up. HbA1c levels decreased in at least $1 \%$ in 96 and increased in eight cases. In the total group HbA1c decreased from $10.1 \pm 1.7$ to $8.4 \pm 1.4 \%$ ( $p \triangleleft 0.01$ ). Those with higher initial values and with oral therapy, had the greatest reductions. At the end of the observation period, 29 patients received one daily dose of InAsBi, 114 two doses and 11 three doses. Two patients had allergy, one systemic and one in the injection site. Conclusions: In this group of diabetic patients with a bad metabolic control, the use of InAsBi was associated with a significant reduction of glycosilated hemoglobin levels (Rev Méd Chile 2007; 135: 1253-60).

(Key w ords: Diabetes mellitus; Hemoglobins; Insulin, aspartic acid (B28))

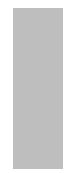

Recibido el 12 de enero, 2007. Aceptado el 20 de abril, 2007.

${ }^{1}$ Unidad de Diabetes y Nutrición, Departamento de Medicina Occidente, Universidad de Chile. Santiago, Chile. ${ }^{2}$ Centro Novocare, Laboratorio Novo Nordisk.

aBioquímico

bEnfermera Universitaria

L a prevalencia de diabetes mellitus (DM), especialmente la tipo 2 , ha incrementado en forma acelerada en el último tiempo $\mathrm{y}$, con ello, los

Correspondencia a: Dr. Carlos Wolff. Casilla 33052, Comeo 9, Santiago, Chile. Fono/Fax: 681 7414. E mail: cwf1255@yahoo.es costos económicos y humanos debidos a su alta morbilidad y mortalidad.

En Chile, la prevalencia de diabetes tipo 2 (DM2), en la población general según la Encuesta Nacional de Salud del año 2003 fue de 4,2\% (4,8\% en hombres y $3,8 \%$ en mujeres), con porcentajes mayores en personas de más de 44 años y sujetos de niveles socioeconómicos más bajos ${ }^{1}$. 
Diversos estudios de intervención han indicado que la morbilidad y mortalidad de la diabetes se puede disminuir significativamente si se logra un control glicémico estricto con terapia insulínica intensificada ${ }^{2-5}$.

La American Diabetes Association (ADA) estima -basándose en el United Kingdom Prospective Diabetes Study- que en los pacientes diabéticos, una elevación de 1\% de la HbA1c aumenta el riesgo de mortalidad en $25 \%$, el de complicaciones crónicas microvasculares en 35\% y el de infarto del miocardio, fatal y no fatal, en $18 \%{ }^{3,4}$.

Estudios epidemiológicos recientes han sugerido que la reducción efectiva de la hiperglicemia postprandial debiera ser un objetivo particularmente importante, porque se asocia a una disminución significativa de los eventos cardiovasculares ${ }^{6-8}$. Sin embargo, en la práctica clínica, el manejo de la diabetes con terapia intensificada de insulina aumenta el riesgo de hipoglicemia. Los análogos de insulina de acción ultrarrápida, como la insulina aspártica y otras, han mejorado esta situación, al permitir la provisión de insulina exógena de manera semejante a la fisiológica ${ }^{9}$.

La insulina aspártica bifásica (InAsBi) es un análogo de insulina premezclada, que contiene $30 \%$ de insulina aspártica soluble de acción rápida y $70 \%$ de insulina aspártica retardada con protamina. El componente soluble de esta forma de presentación de insulina bifásica actúa disminuyendo los niveles glicémicos postprandiales, debido a que se absorbe más rápido y aumenta los niveles séricos de insulina en forma de un pico más alto, en comparación con el componente soluble de la insulina humana premezclada 30/70 convencional ${ }^{10,11}$. Además, el componente de insulina aspártica protamina tiene un perfil de absorción prolongado, que permite un mejor control basal de la glicemia. La insulina aspártica bifásica es de uso actual en diferentes países, aprobada por la Administración de Drogas y Alimentos (FDA) de Estados Unidos de Norteamérica y no se han informado efectos colaterales de importancia.

Cuando se usa en regímenes de inyección múltiple diario bolo - basal, la InAsBi mejora el control glicémico sin provocar hipoglicemias severas ${ }^{12,13}$.

La administración de este tipo de insulina puede tener gran utilidad en pacientes con DM2, que no logran un adecuado control glicémico con hipoglicemiantes orales u otras insulinas.

El propósito de este estudio fue evaluar la eficacia de la InAsBi (Novomix $30^{\circledR}$ ) en una experiencia clínica programada (ECP), en pacientes diabéticos en tratamiento previo con dietoterapia 0 agentes hipoglicemiantes orales, con o sin uso de insulina basal o mezclas, que no hubieran logrado un óptimo control metabólico (HbA1c >7\%).

\section{PACIENTES Y MÉTODOS}

La ECP se programó como un estudio multicéntrico en que cada centro correspondió a una consulta privada de un médico especialista en diabetes de 10 ciudades de Chile. Participaron 66 médicos quienes, entre diciembre de 2005 y marzo de 2006, incorporaron un total de 196 pacientes.

Se consideraron los siguientes criterios de inclusión: portador de diabetes tipo 1 ó 2, con valores de HbA1c inicial mayor de $7 \%$, con 0 sin tratamiento insulínico previo, hombres o mujeres de cualquier edad que hubiesen dado su consentimiento informado y compromiso de participar en el estudio ${ }^{14}$.

Los criterios de exclusión fueron: embarazo, enfermedad concomitante (insuficiencia hepática, cáncer, infección por VIH o hepatitis C), tratamiento con corticoides y complicaciones crónicas avanzadas de la diabetes (retinopatía proliferativa, nefropatía, neuropatía diabética avanzada).

Fueron causa de retiro del estudio: reacciones alérgicas, embarazo, abandono voluntario y el no concurrir a control de HbA1c a las 12 semanas.

Los pacientes que cumplieron los criterios anteriores, pero no se sometieron a un adecuado control de ajuste de dosis de insulina, fueron eliminados del estudio y constituyeron un grupo distinto.

Se consideró éxito del tratamiento la disminución de al menos un punto porcentual de la HbA1c a las 12 semanas de tratamiento con InAsBi.

Una vez seleccionado el paciente por su médico tratante, se le informó del objetivo de la ECP, se realizó una HbA1c basal y fue derivado al «entro de Atención al Paciente Novocare»para su entrenamiento. Allí, una enfermera efectuó una anamnesis de acuerdo a un diseño preestablecido, 
consignando los siguientes antecedentes: edad, género, tipo de diabetes, años de diagnóstico y tratamiento anterior. Además, se programaron sesiones de educación en relación al uso del dispensador (lapicera), autocontrol, manejo de episodios de hipoglicemias, orientación alimentaria y derivación en casos de urgencia.

A cada paciente se le entregó la InAsBi, el dispensador (FlexPen ${ }^{\circledR}$ ), glucómetro, cintas para el autocontrol, formularios para el registro de glicemias, guía de alimentación, instructivo para el reconocimiento de hipo e hiperglicemias y una escala visual de dolor (EVA).

Durante el período de seguimiento, los pacientes fueron contactados telefónicamente por la enfermera una vez a la semana para evaluar el autocontrol, informándole posteriormente al médico tratante el estado del paciente.

Al finalizar la observación (12 semanas), se realizó la HbA1c final y el paciente respondió una encuesta telefónica de satisfacción usuaria que evaluó: facilidad en la aplicación, tiempo del procedimiento, percepción del dolor y seguridad en la selección de las dosis con el dispensador.

A los pacientes que antes de ingresar a la ECP se encontraban sólo con medidas no farmacológicas o drogas hipoglicemiantes orales (DHO), se les recomendó iniciar el tratamiento con una sola inyección de $12 \mathrm{U}$ de InAsBi antes de la cena. A los que previamente recibían tratamiento insulínico, se les indicó InAsBi en igual número de unidades. Aquellos pacientes que requerían más de $30 \mathrm{U}$ de InAsBi, fueron instruidos para fraccionarla en 2 dosis iguales, antes del desayuno y cena. El ajuste de dosis semanal de insulina recomendada según el nivel de glicemia se presenta en la Tabla 1.

La necesidad de indicar una segunda dosis de insulina dependió del control de la glicemia precena. Cuando ello fue necesario, se distribuyó $2 / 3$ de la dosis en la mañana y $1 / 3$ en la noche. En algunos casos -cuando la glicemia precena permanecía alta a pesar del ajuste de la dosis matinal- fue necesario agregar una tercera dosis de InAsBi antes del almuerzo.

No se incluyeron en la investigación 18 (9,2\%) sujetos por no tener estudio completo. Un paciente $(0,5 \%)$ se retiró por reacción alérgica sistémica leve.

Veintitrés individuos se separaron en un grupo aparte, porque fueron incapaces de realizar autocontrol y ajustar las dosis de insulina. Los resultados obtenidos en este grupo se emplearon para evaluar el efecto del adecuado ajuste de dosis de insulina en el éxito del tratamiento con InAsBi. Por lo tanto, el grupo de estudio quedó finalmente constituido por 154 pacientes, 84 mujeres y 70 hombres. Ciento cuarenta y dos eran portadores de DM2 y 8 de DM1. La edad del grupo fluctuó entre 15 y 84 años, y el promedio $(\mathrm{X} \pm \mathrm{DE})$ fue de $58,6 \pm 12,4$ años.

La distribución de los pacientes según el tratamiento previo se muestra en la Tabla 2, observándose que $48,1 \%$ se encontraba sin tratamiento insulínico.

\section{Tabla 1. Recomendación de ajuste de dosis semanal de insulina aspártica bifásica según glicemia}

\begin{tabular}{|lc|}
\hline Glicemia $(\mathrm{mg} / \mathrm{dl})$ & Dosis de insulina aspártica \\
\hline$<80$ & Disminuir $2 \mathrm{U}$ \\
$80-110$ & Mantener dosis \\
$111-140$ & Aumentar $2 \mathrm{U}$ \\
$141-180$ & Aumentar $4 \mathrm{U}$ \\
$>180$ & Aumentar $6 \mathrm{U}$ \\
\hline
\end{tabular}

Tabla 2. D istribución de pacientes según tratamiento previo

\begin{tabular}{|lrr|}
\hline Tratamiento previo & $\mathrm{n}$ & $\%$ \\
\hline Dieta y ejercicio & 34 & 22,1 \\
DHO & 40 & 26,0 \\
I. glargina + I. cristalina & 5 & 3,2 \\
Mezcla 30/70 & 2 & 1,3 \\
I. NPH sola o asociada a I. cristalina, o asociada a DHO & 73 & 47,4 \\
\hline
\end{tabular}

$\mathrm{DHO}=$ drogas hipoglicemiantes orales, $\mathrm{I}=$ insulina, NPH= (Neutral Protamine Hagedorn). 
Se consideró como antigüedad de la enfermedad el tiempo transcurrido entre la fecha del diagnóstico de la DM y el inicio del estudio. Cien pacientes (65\%) tenían más de 10 años de evolución de la diabetes. El intervalo [Min; Max] de antigüedad de la diabetes fue [0-42] años, la mediana de 11,0 años y el promedio $(\mathrm{X} \pm \mathrm{DE})$ $14,0 \pm 9,0$ años.

La HbA1c fue evaluada en muestras de sangre capilar en distintos laboratorios, pero todos pertenecientes a la red de control de calidad del Instituto de Salud Pública de Chile y por el procedimiento inmunoquímico, que utiliza anticuerpos monoclonales dirigidos a secuencias aminoacídicas específicas de la HbA1c (DCA 2000, Bayer). El método presenta valores de referencia entre $4 \%$ y $6 \%$.

El análisis de los resultados se realizó mediante prueba $t$ de Student pareada o mediante prueba no paramétrica de Mann Whitney, según si la distribución fue normal o no. Se utilizó el programa de análisis estadístico Medcalc.

\section{RESULTADOS}

Los resultados obtenidos con el uso de InAsBi en los pacientes DM1 fueron similares a los logrados en los DM2, por lo cual se analizaron en conjunto.
Noventa y seis de los 154 (62\%) pacientes estudiados disminuyeron a lo menos un punto porcentual la HbA1c a las 12 semanas de tratamiento, 56 (36,4\%) permanecieron con una HbA1c semejante a la del inicio y sólo $8(5,2 \%)$ la aumentaron (Figura 1).

En el total de los casos estudiados se observó una diferencia estadísticamente significativa entre la HbA1c inicial y la final ( $p<0,0001$ ) (Tabla 3). Los pacientes con valores iniciales de HbA1c más altos, presentaron una disminución más acentuada.

Veinticinco (16,2\%) pacientes bajaron la HbA1c a un valor menor o igual a 7\%, diecisiete redujeron en 2 o más puntos la hemoglobina glicosilada inicial.

Se encontratron diferencias estadísticamente significativas $(p<0,001)$ en los cambios producidos en la HbA1c, según si previo al inicio del tratamiento con InAsBi, el paciente se encontraba con dieta o DHO o en terapia con otras insulinas (Tabla 4).

Se observaron diferencias estadísticamente significativas ( $p<0,0001)$ en la variación experimentada por el valor de la HbA1c a las 12 semanas de tratamiento, en relación al ajuste de dosis. Para ello se compararon los resultados de la HbA1c de los 23 casos eliminados del estudio por mal ajuste de dosis, con los 154 pacientes que cumplieron el protocolo y finalizaron la investigación (Tabla 5).
Figura 1. Número de pacientes según cambio en el valor de la HbA1c después de 12 semanas de tratamiento.

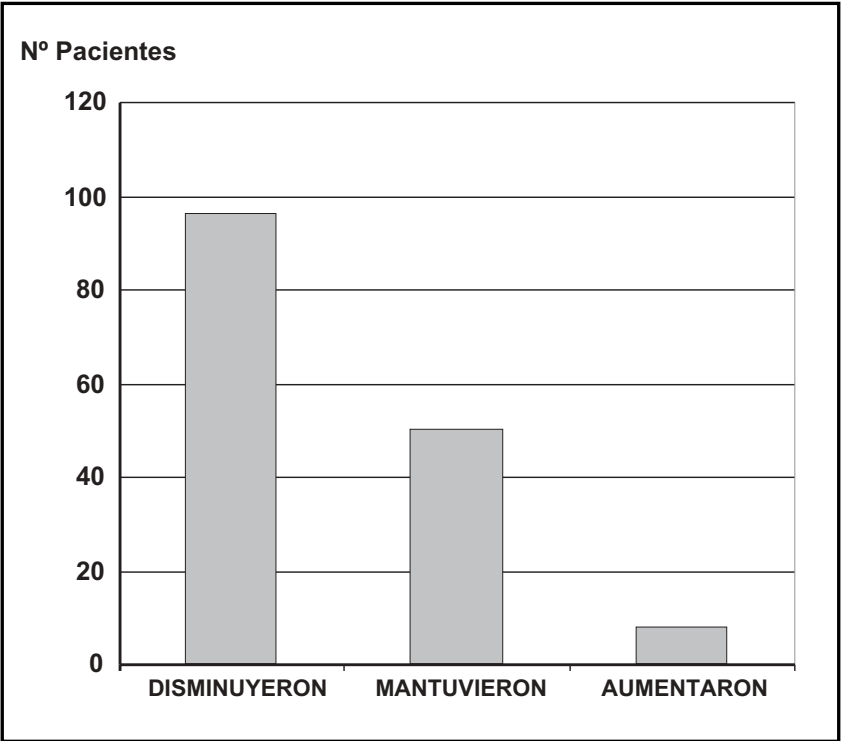


Tabla 3. Variación en la H bAlc a las 12 semanas de tratamiento

\begin{tabular}{|lccc|} 
& Inicial & HbA1c & Variación \\
\hline$[$ Min; Max $]$ & {$[7,1 ; 14,3]$} & {$[5,4 ; 12,6]$} & {$[-7,7 ; 3,2]$} \\
Promedio \pm DE & $10,1 \pm 1,7$ & $8,4 \pm 1,4$ & $1,7 \pm 1,8$ \\
Mediana* & 10,0 & 8,3 & 1,3 \\
\hline
\end{tabular}

$* \mathrm{p}<0,0001$.

Tabla 4. Variación de H bAlc según tratamiento previo

\begin{tabular}{|lcc|}
\hline & Dieta o DHO & Otras insulinas \\
\hline $\mathrm{n}$ & 74 & 80 \\
[Min; Max $]$ & {$[1,6 ;-7,7]$} & {$[3,2--6,0]$} \\
Mediana* & $-2,1$ & $-0,9$ \\
Promedio \pm DE & $-2,2 \pm 2,0$ & $-1,2 \pm 1,6$ \\
\hline
\end{tabular}

$*_{p}<0,001$.

Cuando hubo un ajuste deficiente de dosis de insulina, la mayoría de los pacientes presentó un aumento de la HbA1c.

Con el objeto de obtener un buen control de la glicemia durante el tratamiento, los pacientes requirieron un incremento estadísticamente significativo $(p<0,001)$ de la dosis diaria promedio de insulina (Tabla 6).

No hubo diferencias estadísticamente significativas en el requerimiento insulínico respecto al tratamiento que recibían previamente los pacientes.

Veintinueve sujetos (18,8\%), al finalizar la ECP, recibían una inyección diaria de insulina; de ellos,

\section{Tabla 5. Variación de la H bAlc} según el control de ajuste de dosis

\begin{tabular}{|lcc|}
\hline & Malo & Bueno \\
\hline $\mathrm{n}$ & 23 & 154 \\
{$[$ Min; Max $]$} & {$[3,0 ;-2,9]$} & {$[3,2 ;-7,7]$} \\
Mediana* & 0,1 & $-1,3$ \\
Promedio \pm DE & $0,2 \pm 1,3$ & $-1,7 \pm 1,8$ \\
\hline
\end{tabular}

$*_{p}<0,0001$.

23 se la administraban en forma matinal y 6 precena. Ciento catorce (74\%) individuos requirieron dos dosis diarias (matinal y nocturna) y 11 $(7,1 \%)$ tres inyecciones: matinal, prealmuerzo y precena.

Los niveles de HbA1c para lograr un buen control glicémico, fueron similares e independientes del número de inyecciones de InAsBi administradas.

Un paciente desarrolló una alergia sistémica leve que el médico tratante consideró causal para suspender el tratamiento. Un segundo caso presentó una reacción local en el punto de inyección

Tabla 6. Variación en la dosis diaria promedio de insulina aspártica bifásica (unidades/día)

\begin{tabular}{|lccc|}
\hline & Inicial & Final & Variación \\
\hline$[$ Min; Max $]$ & {$[4 ; 86]$} & {$[5 ; 120]$} & {$[39 ; 90]$} \\
Mediana* & 12 & 32 & 16 \\
Promedio \pm DE & $20,8 \pm 15,6$ & $38,2 \pm 18,6$ & $17,4 \pm 16,7$ \\
\hline
\end{tabular}

$*_{p}<0,001$. 
una vez completado el período de observación, por lo que fue incorporado al análisis de los datos. No se informó de episodios de hipoglicemias de grado moderado o severo.

No existieron diferencias estadísticamente significativas en los cambios de la HbA1c según el nivel educacional (Tabla 7).

El 95\% de los pacientes encontró que la aplicación de InAsBi con el dispensador Flexpen ${ }^{\circledR}$ fue muy fácil o fácil. El $90 \%$ de los casos informó no haber tenido molestias con la inyección de insulina.

\section{Discusión}

En esta experiencia clínica se observó que un alto porcentaje (62\%) de pacientes con diabetes mal controlada, presentaron una reducción de uno 0 más puntos porcentuales de la HbA1c al ser tratados con insulina aspártica bifásica (Novomix $30^{\circledR}$ ) durante 12 semanas. Es posible que los resultados pudieran haber sido aún mejores si el período de observación hubiese sido mayor, como lo han hecho notar otros autores en ensayos clínicos controlados ${ }^{15}$. Sin embargo, se eligió este período relativamente breve de tratamiento, por cuanto está ampliamente demostrado que HbA1c expresa el grado de control metabólico de los últimos 3 meses. La asociación de metformina con InAsBi permite obtener mejores resultados, como ha sido demostrado por Kvapil y $\mathrm{col}^{16}$. Los pacientes con valores más altos presentaron disminución más acentuada de la hemoglobina glicosilada, hecho confirmado en otros estudios con terapia insulínica bifásica ${ }^{13}$.
Llama la atención que cuando hay un inadecuado ajuste de las dosis de insulina, en la mayoría de los casos hay un aumento de la HbA1c, lo que reafirma que todo paciente diabético en insulinoterapia requiere de un fuerte apoyo de enfermería para educación y adecuado manejo del autocontrol.

El mayor éxito obtenido en pacientes diabéticos con fracaso secundario a drogas orales, comparados con aquellos que ya estaban recibiendo insulina, se explicaría porque estos últimos ya habían mejorado su control metabólico y, por lo tanto, reducido sus niveles de HbA1c.

Si bien -según el protocolo- todos los pacientes iniciaron el tratamiento con una dosis de InAsBi, al correr el tiempo y de acuerdo al automonitoreo, $18,8 \%$ se mantuvo con una inyección al día, mientras $74 \%$ requirió de dos dosis y sólo 7,1\% necesitó de 3 aplicaciones. Lo señalado es de toda lógica, de acuerdo a la farmacocinética y farmacodinamia de esta insulina ${ }^{10}$, en que el componente de aspártico retardado con protamina tiene una acción que no va más allá de 18-20 h y el paciente requiere por ello, de una segunda dosis para cubrir las $24 \mathrm{~h}$. Casos más infrecuentes, que presentaban hiperglicemias postalmuerzo, necesitaron de una tercera inyección antes de esta ingesta. Los hechos que comentamos han sido también publicados por otros autores, tanto en DM2 ${ }^{15}$ como DM1 ${ }^{17}$.

El no haber observado casos de hipoglicemias severas, constituye una ventaja comparativa del uso del InAsBi frente a otras insulinas, atribuible a la especial farmacocinética del producto ${ }^{9,18}$.

Dado que este estudio se realizó como una experiencia clínica programada y no como un

Tabla 7. Cambio en el valor de la H bAlc después de 12 semanas de tratamiento con insulina aspártica bifásica según nivel educacional

\begin{tabular}{|lccc|}
\hline HbA1c & Básico & $\begin{array}{c}\text { Nivel educacional } \\
\text { Medio }\end{array}$ & Superior \\
\hline $\mathrm{n}$ & 37 & 71 & 43 \\
[Min; Max $]$ & {$[-6,9 ; 0,7]$} & {$[-7,0 ; 2,0]$} & {$[-7,7 ; 3,2]$} \\
Mediana & $-1,0$ & $-1,4$ & $-1,3$ \\
Promedio \pm DE & $-1,7 \pm 1,8$ & $-1,6 \pm 1,9$ & $-1,7 \pm 1,8$ \\
\hline
\end{tabular}


ensayo clínico, no se analizaron otras variables, tales como: el peso del paciente, las glicemias postprandiales u otros parámetros clínicos, porque estaban fuera del objetivo de esta investigación. Es sabido que el empleo de insulina provoca aumento de peso, el que en una observación corta, como la de este trabajo, no es relevante.

El hecho que el éxito del tratamiento fuera independiente del nivel educacional, puede ser atribuido a que esta experiencia clínica fue diseñada, con un fuerte apoyo educativo y contactos telefónicos frecuentes y programados.

Es posible que un factor importante en los resultados obtenidos se haya debido al uso de un dispositivo seguro y de fácil administración (FlexPen $\left.{ }^{\circledR}\right)$, que se reflejó en el alto porcentaje de satisfacción del usuario, lo que favoreció la adhesión al tratamiento.

\section{REFERENCIAS}

1. http://epi.minsal.cl/epi/htm/invet/ENS/informe Final ENS.pdf

2. Diabetes Control and Complication Trial (DCCT) ReSEARCH Group. The absence of a glycemic threshold for the development of long-term complications: the perspective of the Diabetes Control and Complications Trial. Diabetes 1996; 45: 1289-98.

3. American Diabetes Association. Implications of the United Kingdom Prospective Diabetes Study. Diabetes Care 2003; 26 (Suppl 1): S28-S32.

4. Stratton IM, Adier AI, Neil HA, Matthews DR, Manley SE, Cull CA et al. Association of glycaemia with macrovascular and microvascular complications of type 2 diabetes (UKPDS 35): prospective observational study. BMJ 2000; 321: 405-12.

5. KLEIN R. Hyperglycaemia and microvascular and macrovascular disease in diabetes. Diabetes Care 1995; 18: 258-71.

6. DECODE Study Group. Glucose tolerance and cardiovascular mortality: Comparison of fasting and 2-hour diagnostic criteria. Arch Intern Med 2001; 161: 397-405.

7. Cerielo A, Hanefeld M, Leiter L, Monnier L, Moses A, Owens D ET AL. Postprandial glucose regulation
En conclusión, este estudio clínico muestra, por una parte, la eficacia del uso de insulina aspártica bifásica en diabéticos en mal control metabólico con terapias orales u otros tipos de insulina y, por otra, su flexibilidad al poder iniciar el tratamiento con una dosis diaria, para aumentar a 2 ó 3 inyecciones, si fuera necesario, e intensificar su dosificación según los autocontroles. Todo ello de manera segura y con bajo riesgo de hipoglicemias.

Agradecimientos

Agradecemos al Laboratorio Novo Nordisk haber aportado la insulina aspártica bifásica empleada para el tratamiento de los pacientes, lo que hizo posible este estudio, sin participar en el diseño del estudio, en el análisis de los resultados ni en la redacción del manuscrito.

and diabetic complications. Arch Intern Med 2004; 164: 2090-5.

8. Hanefeld M, Fischer S, Julus U, Schuize J, SchwaneBECK U, SCHMECHeL $H$ ET AL Risk factors for myocardial infarction and death in newly detected NIDDM: the Diabetes Intervention Study, 11year follow-up. Diabetologia 1996; 39: 1577-83.

9. Home PD, Lindholm A, Hy山eberg B, Round P. Improved glycemic control with insulin aspart: a multicenter randomized double-blind crossover trial in type 1 diabetic patients. UK Insulin Aspart Study Group. Diabetes Care 1998; 21: 1904-9.

10. Weyer C, Heinemann L, Heise T. Insulin aspart in 30/70 premixed formulation. Pharmacodynamic properties of a rapid acting insulin analog in stable mixture. Diabetes 1997; 20: 1612-4.

11. Hermansen K, Colombo M, Storgaard H, OstergaARD A, Kolendorf K, Madsbad S. Improved postprandial glycemic control with biphasic insulin aspart relative to biphasic insulin lispro and biphasic human insulin in patients with type 2 diabetes. Diabetes Care 2002; 25: 883-8.

12. Lngvay I, Kaloyanova PF, Raskin P. Newly diagnosed patients with type 2 diabetes mellitus are satisfied with insulin treatment. Diabetologia 2006; 49 (Suppl 1): Abs 868.

13. Raskin P, Auen E, Holandere P, Lewin A, Gabbay RA, Hu $P$ et al. INITIATE Study Group. Initiating 
insulin therapy in type 2 Diabetes: a comparison of biphasic and basal insulin analogs. Diabetes Care 2005; 28: 260-5.

14. Declaration of Helsinki. Recommendations guiding medical physicians in biomedical research involving human subjects. JAMA 1997; 277: 925-6.

15. Garber AJ, Wahlen J, Wahl T, Bressier P, Braceras R, Auen E et AL. Attainment of glycaemic goals in type 2 diabetes with once-twice, or thrice-daily dosing with biphasic insulin aspart 70/30 (The 12-3 study). Diabetes Obes Metab 2006; 8: 58-66.

16. Kvapil M, Swatko A, Hilberg C, Shestakova M. Biphasic insulin aspart 30 plus metformin: an effective combination in type 2 diabetes. Diabetes Obes Metab 2006; 8: 39-48.

17. Boenm BO, Home PD, Behrend C, Kamp NM, LiNDHOLM A. Premixed insulin aspart 30 versus premixed human insulin 30/70 twice daily: a randomized trial in type 1 and type 2 diabetic patients. Diabetic Medicine 2002; 19: 393-9.

18. Luzio S, Dunseath G, Peter R, Pauvaday V, Owens DR. Comparison of the pharmacokinetics and pharmacodynamics of biphasic insulin aspart and insulin glargine in people with type 2 diabetes. Diabetologia 2006; 49: 1163-8. 